\title{
Corrigendum: Elucidation of the Signatures of Proteasome-Catalysed Peptide Splicing
}

\author{
Wayne Paes ${ }^{1 *}$, German Leonov ${ }^{2}$, Thomas Partridge ${ }^{1}$, Annalisa Nicastri ${ }^{3}$, Nicola Ternette ${ }^{3}$ \\ and Persephone Borrow ${ }^{1 *}$ \\ ${ }^{1}$ Nuffield Department of Clinical Medicine, University of Oxford, Oxford, United Kingdom, ${ }^{2}$ York Cross-Disciplinary Centre for \\ Systems Analysis, University of York, York, United Kingdom, ${ }^{3}$ Nuffield Department of Clinical Medicine, The Jenner Institute, \\ University of Oxford, Oxford, United Kingdom
}

Keywords: peptide splicing, proteasome, splicing mechanism, antigen processing, peptide epitopes

\section{A Corrigendum on}

Elucidation of the Signatures of Proteasome-Catalyzed Peptide Splicing

By Paes W, Leonov G, Partridge T, Nicastri A, Ternette $N$ and Borrow P (2020). Front. Immunol. 11:563800. doi: 10.3389/fimmu.2020.563800

\section{OPEN ACCESS}

Edited and reviewed by: Peter M. Van Endert, Institut National de la Santé et de la Recherche Médicale (INSERM),

France

${ }^{*}$ Correspondence: Wayne Paes

wayne.paes@ndm.ox.ac.uk Persephone Borrow persephone.borrow@ndm.ox.ac.uk

Specialty section:

This article was submitted to Antigen Presenting Cell Biology, a section of the journal

Frontiers in Immunology

Received: 07 August 2021

Accepted: 25 August 2021 Published: 23 September 2021

Citation:

Paes W, Leonov G, Partridge T, Nicastri $A$, Ternette $N$ and Borrow $P$

(2021) Corrigendum: Elucidation of the Signatures of ProteasomeCatalysed Peptide Splicing.

Front. Immunol. 12:755002. doi: 10.3389/fimmu.2021.755002
There were three minor errors in our original article.

1) The Materials and Methods section required additional detail about the search strategy implemented in Peaks and the discovery workflow to facilitate replication of the methodology. Two extra sentences have been added to expand on this, and a third sentence describing a step that was used in our previous HLA-I discovery workflow for spliced peptides, but that is not required in the interrogation of datasets derived from polypeptide digests, has been removed.

A correction has been made to Materials and Methods, Discovery workflow for identification of non-spliced and spliced peptides, paragraphs 1,2 and 3:

Paragraph 1: "PEAKS de novo assisted sequencing was implemented for the assignment of nonspliced peptides derived from individual polypeptide sequences following proteasomal digest of each of the 25 precursor substrates, and no PTMs were included in the de novo search space".

Paragraph 2: "Therefore, for DNUPs containing a total of ' $n$ ' leucine residues, all permutations $\left(2^{\mathrm{n}}\right)$ of L/I variants were computed prior to in silico splicing - e.g. for de novo sequence LTSLTLKE originating from polypeptide precursor LTSLVRRATLKENEQIPK, $2^{3}$ combinations of the original de novo sequence would be computed (LTSLTLKE, LTSITLKE, ITSLTLKE, LTSLTIKE, LTSITIKE, ITSLTIKE, ITSITLKE, ITSITIKE) and each sequence input to the splicing algorithm".

Paragraph 3: "The $(n-1)^{\text {th }}$ fragment was first scanned for a contiguous match across the polypeptide, and when found, its corresponding splice partner fragment was scanned for a contiguous match within the remainder of the polypeptide sequence. Due to the lack of an applied false discovery rate (FDR) for identification of spliced peptides from de novo sequencing of LC-MS/MS spectra that were not matched to a pre-defined database, very short spliced peptide sequences (5-7 aa) were omitted from analysis, and only sequences with a length of 8 aa or greater were considered. Trans-spliced peptides were omitted from the analysis". 
2) The sequence of precursor polypeptide PP13 was erroneously given in Table 1 as LQPQLIHLYYFDCFSESAIRNA, but this peptide in fact ended in ' $\mathrm{K}$ ' and not 'NA'. The PP13 sequence has now been amended to LQPQLIHLYYFDCFSESAIRK; and overall amino acid frequencies within polypeptide precursors in Table $\mathbf{2}$ have been adjusted to reflect this minor change. The corrected Table 1 and Table 2 appear below.

3) In Supplementary Table 2, eight of the original undigested precursor polypeptide sequences were accidentally included in the non-spliced lists (PP7, PP8, PP16, PP17, PP19, PP20, PP21, PP25). These have now been removed. Four spliced peptides (EGCPMVVKF, ALIKPLPSV, FIRNLSFKCS, SFKCSEDDLKTVFAQFGAK) were also erroneously present in the non-spliced lists. Two of them have now been removed as they were 1-mer fusions (FIRNLSFKCS, SFKCSEDDLKTVFAQFGAK) which we did not consider in the manuscript, while the 2 cis-spliced peptides (EGCPMVVKF, ALIKPLPSV) have been added to the spliced peptide lists. The corrected Supplementary Material can be accessed via the link below.

Values in the sentence of the results text stating the total number and percentages of non-spliced and spliced peptides have been amended accordingly. The corrected sentence in the Results, The Relative Proportions of Unique Non-spliced and Cis-Spliced Peptides Generated by the Constitutive Proteasome Are Dependent on Precursor Peptide Length is now as follows:

"Overall, we observed a total of 1,200 unique non-spliced (72.9\%) and 446 cis-spliced (27.1\%) peptides (Figure 1A)".
The relevant panels of Figure $\mathbf{1}$ and Supplementary Figures 1, 2 have also been amended, and figure legends have been revised as needed. The corrected Figure $\mathbf{1}$ and caption appear below.

"(A) Proportion of unique spliced and non-spliced peptides following a $2 \mathrm{~h}$ in vitro digestion of 25 self- and HIV-1-derived polypeptides (Table 1) by the constitutive proteasome. Proportions of spliced and non-spliced peptides within all unique peptides $(n=1,646)$, unique peptides originating from only the 13 longest polypeptide substrates $(n=1,331)$ and unique peptides originating from only the 12 shortest polypeptide precursors $(n=315)$ are shown".

Given the large number of peptides observed overall, the changes made to the peptide lists in Supplementary Tables 1, 2 have not had any impact on the conclusions drawn from the data, and the revised figure panels are not discernibly different from the originals. Importantly, none of these 12 peptides were used for any of the analyses in the manuscript from Figure 2 onwards, as they all contained terminal amino acids of the polypeptide precursors.

In addition to these minor errors in the original version of our article, there was also an omission: the project accession numbers for the mass spectrometry datasets from the control undigested precursor substrates were not included in the Data Availability Statement. These have now been added.

The Data Availability Statement is now as follows:

"Mass spectrometry proteomics datasets have been deposited to the ProteomeXchange Consortium via the PRIDE partner repository (https://www.ebi.ac.uk/pride) with project accession numbers PXD025893 (for undigested precursor substrates) and PXD021339 (for proteasomal digests of precursor substrates).

TABLE 1 | Synthetic polypeptides subjected to in vitro proteasomal digestion.

\begin{tabular}{|c|c|c|c|}
\hline Polypeptide ID & Protein ID & Sequence & Length (aa) \\
\hline PP1 & sORF-encoded polypeptide: APITD1 & SSCLPCPLSFEKFK & 14 \\
\hline PP2 & Q92879: CUGBP Elav-like family member 1 & EGCSSPMWKFADTQK & 16 \\
\hline PP3 & Q5CZC0: Fibrous sheath-interacting protein 2 & LVSIQKSIVSRSPIMIDQ & 18 \\
\hline PP4 & P15882: N-chimerin & LTSLVRRATLKENEQIPK & 18 \\
\hline PP5 & sORF-encoded polypeptide: CD81 & LPRFESRVCGHSLPSCTCP & 19 \\
\hline PP6 & HIV-1 CH529 enr: Vif & DQLIHLYYFDCFSESAIRK & 19 \\
\hline PP7 & P37275: Zinc finger E-box-binding homeobox 1 & SLIPVNGRPRTGLKTSQCS & 19 \\
\hline PP8 & Q8TDU5: Putative vomeronasal receptor-like protein 4 & HLPLIHILLLFTQAILVSS & 19 \\
\hline PP9 & HIV-1 IIIB: Vif & ALIKPKQIKPPLPSVRKLTE & 20 \\
\hline PP10 & HIV-1 CH390 enr: Vif & TADQLIHLYYFDCFSESAIRK & 21 \\
\hline PP11 & Q9NW13: RNA-binding protein 28 & IRNLSFKCSEDDLKTVFAQFGA & 22 \\
\hline PP12 & HIV-1 CH945 enr: Vif & LADQLIHLYHFDCFTESAIRNA & 22 \\
\hline PP13 & HIV-1 CH945 enr: Vif mutant & LQPQLIHLYYFDCFSESAIRK & 22 \\
\hline PP14 & HIV-1 NL4-3: Gag & FGEETITPSQKQEPIDKELYPLA & 23 \\
\hline PP15 & HIV-1 NL4-3: Gag & AAMQMLKETINEEAAEWDRLHPVHA & 25 \\
\hline PP16 & P46013: Antigen Kl-67 & KSWADWKLGAKQTQTKVIKHGPQR & 25 \\
\hline PP17 & Q00887:Pregnancy-specific beta-1-glycoprotein 9 & EMTDLYHYIISYIVDGKIIIYGPAY & 25 \\
\hline PP18 & HIV-1 IIIB: Cryptic ORF & VAAPRLLPCALQQAESCVERSPLALLS & 27 \\
\hline PP19 & P47989: Xanthine dehydrogenase/oxidase & PRKQLRFEGERVTWIQASTLKELLDLK & 27 \\
\hline PP20 & Q92608: Dedicator of cytokinesis protein 2 & YLDTSSRGEQCEPILRTLKALEYVFKFI & 28 \\
\hline PP21 & Q5T7P8: Synaptotagmin-6 & KLKDPSTLGFLEAAVKISHTSPDIPAEVQM & 30 \\
\hline PP22 & HIV-1 NL4-3: Pol & AELELAENREILKEPVHGAYYDPSKDLIAEL & 31 \\
\hline PP23 & Q14894: Thiomorphine-carboxylate dehydrogenase & ALTTKLVTFYEDRGITSWPSHQATVLLFEPSNG & 34 \\
\hline PP24 & HIV-1 NL4-3: Pol & IRKVLFLDGIDKAQEEHEKYHSNWRAMASDFNLPPWAKEIVAS & 44 \\
\hline PP25 & Q9H8V3: Protein ECT2 & KALKTIKIMEVPVIKIKESCPGKSDEKLIKSVINMDIKVGFVKMESV & 47 \\
\hline
\end{tabular}


TABLE 2 | Amino acid frequencies within synthetic polypeptides and combined HIV-1 and UniProt human proteomes.

Amino acid $^{\text {a }}$

Frequency in polypeptides (\%)

Frequency in combined HIV-1/human proteomes (\%)

\begin{tabular}{|c|c|c|}
\hline$A$ & 7.16 & 8.25 \\
\hline C & 2.65 & 1.37 \\
\hline D & 4.56 & 5.45 \\
\hline$E$ & 7.49 & 6.75 \\
\hline $\mathrm{F}$ & 4.23 & 3.86 \\
\hline$G$ & 3.09 & 7.07 \\
\hline $\mathrm{H}$ & 2.76 & 2.27 \\
\hline 1 & 7.65 & 5.96 \\
\hline $\mathrm{K}$ & 8.31 & 5.84 \\
\hline $\mathrm{L}$ & 11.24 & 9.66 \\
\hline$M$ & 1.63 & 2.42 \\
\hline $\mathrm{N}$ & 1.63 & 4.06 \\
\hline$P$ & 6.19 & 4.7 \\
\hline$Q$ & 4.40 & 3.93 \\
\hline $\mathrm{R}$ & 4.23 & 5.53 \\
\hline$S$ & 8.31 & 6.56 \\
\hline $\mathrm{T}$ & 4.89 & 5.34 \\
\hline V & 5.86 & 6.87 \\
\hline W & 0.65 & 1.08 \\
\hline$Y$ & 3.09 & 2.92 \\
\hline
\end{tabular}

${ }^{a}$ Amino acids are denoted using single letter code.
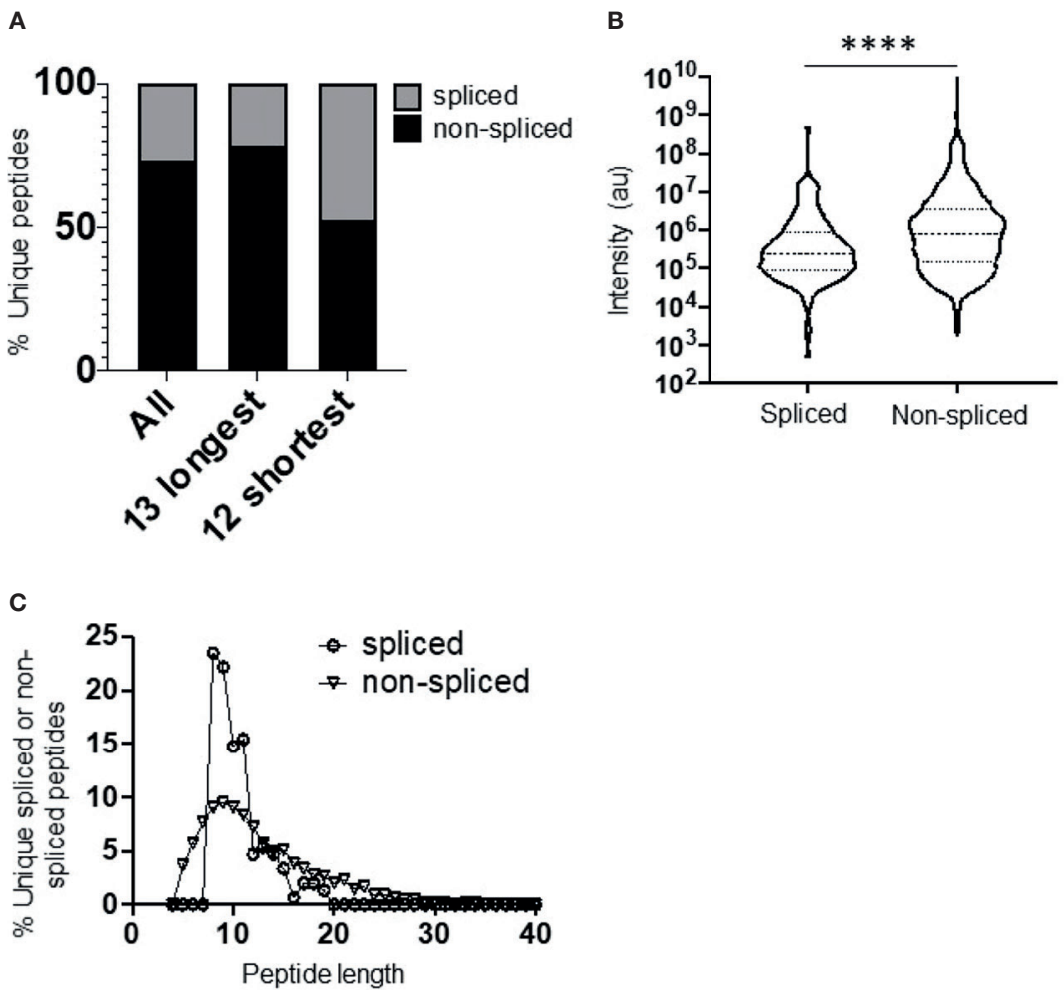

FIGURE 1 | Diversity, abundance, and peptide length distribution of proteasome-derived spliced and non-spliced peptides. (A) Proportion of unique spliced and non-spliced peptides following a $2 \mathrm{~h}$ in vitro digestion of 25 self- and HIV-1-derived polypeptides (Table 1) by the constitutive proteasome. Proportions of spliced and non-spliced peptides within all unique peptides $(n=1,656)$, unique peptides originating from only the 13 longest polypeptide substrates $(n=1,337)$ and unique peptides originating from only the 12 shortest polypeptide precursors ( $n=319)$ are shown. (B) Violin plots showing abundance of all unique spliced and non-spliced peptides as measured by LC-MS/MS intensity values. Median and quartile abundance values are indicated. A non-parametric unpaired Mann-Whitney t-test was used to determine whether abundance values differed between groups. ${ }^{* \star \star *} \mathrm{P}<0.0001$. (C) Length distributions of unique spliced ( $\left.\mathrm{C}=135\right)$ and non-spliced $(\mathrm{n}=$ 900) peptides generated from within polypeptide substrates following a $2 \mathrm{~h}$ proteasomal digest. 
Computer scripts have likewise also been made privately available in GitHub and are available on request".

The authors apologize for these errors and omissions, and state that they do not change the scientific conclusions of the article in any way. The original article has been updated.

\section{SUPPLEMENTARY MATERIAL}

The Supplementary Material for this article can be found online at: https://www.frontiersin.org/articles/10.3389/fimmu.2021. 755002/full\#supplementary-material
Publisher's Note: All claims expressed in this article are solely those of the authors and do not necessarily represent those of their affiliated organizations, or those of the publisher, the editors and the reviewers. Any product that may be evaluated in this article, or claim that may be made by its manufacturer, is not guaranteed or endorsed by the publisher.

Copyright (๑) 2021 Paes, Leonov, Partridge, Nicastri, Ternette and Borrow. This is an open-access article distributed under the terms of the Creative Commons Attribution License (CC BY). The use, distribution or reproduction in other forums is permitted, provided the original author(s) and the copyright owner(s) are credited and that the original publication in this journal is cited, in accordance with accepted academic practice. No use, distribution or reproduction is permitted which does not comply with these terms. 